\title{
Unidad de endoscopia: parámetros mínimos de calidad, ergonomía, seguridad intra y extrahospitalaria, recuperación y radioprotección
}

\author{
Yolanda Zamorano-Orozco* \\ Servicio de endoscopia y Fisiología Digestiva, Hospital General Regional No. 1 "Dr Carlos Mac Gregor Sánchez Navarro", Ciudad de México, México
}

Resumen:

Los estándares de calidad en la unidad de Endoscopia, medidas de protección al personal que la conforman, están normados a nivel internacional, con todos los requerimientos de seguridad intra y extrahospitalaria, en lo que respecta a ergonomía, radio protección y en la ejecución del estudio endoscópico.

Palabras clave: Calidad. Seguridad intra y extrahospitalaria. Ergonomía. Radioprotección.

El estudio endoscópico es un procedimiento diagnóstico y terapéutico considerado una rutina cotidiana del médico endoscopista, los procedimientos se podrán realizar en unidades de corta estancia, que cuenten con la infraestructura necesaria para la realización de estos, como lo es: monitor de signos vitales, máquina de anestesia, tomas de oxígeno y aspiración, carro rojo, contenedores de residuos biológicamente peligrosos, unidad de lavado (área específica). En el foro de la AGA se presentó un Simposio de los parámetros básicos de calidad en lo que respecta al procedimiento endoscópico especifico, dichos parámetros básico se especifican en GIE:2015 Vol. 81 (Quality Indicators for all endoscopic procedures) ${ }^{1}$, en lo que respecta al procedimiento endoscópi$\mathrm{co}$, desde endoscopista que la realiza, que cuente con las competencias y certificación que acredite una buena praxis, a la estructura e infraestructura de la sala de procedimientos endoscópicos, la entrega oportuna de resultados y seguimiento del resultado de histopatológico, sedación por personal capacitado y certificado, consentimiento informado. Los resultados y estadística de las complicaciones del procedimiento y sobre todo el costo.
Uno de los parámetros de mayor énfasis es que al cumplir con todas las normas de calidad, esto tiene un impacto positivo en la reducción de los costos y días de estancia intrahospitalaria.

En patologías especiales como lo es el esófago de Barrett, su identificación y seguimiento de acuerdo con las guías de práctica clínica y revisiones de experto, en los pacientes cirróticos el uso correcto de antimicrobianos, seguimiento y evaluación de los pacientes con hipertensión portal (várices esofágicas y gástricas) el protocolo de biopsias en los pacientes con enfermedad celiaca. Los resultados van directamente relacionados de acuerdo con la presentación de calidad de la Universidad de Minnesota presentado por John Allen², donde los resultados tienen relación directa con el cumplimiento de los estándares de calidad, seguridad, accesibilidad y experiencia, el impacto positivo es la disminución en los costos por cada procedimiento, baja tasa de complicaciones y repetición de los exámenes de endoscopia y el impacto en los intervalos de supervivencia.

También en el foro de la ASGE, se presentaron diversos foros de calidad en colonoscopia, ultrasonido endoscópico y endoscopia de las vías biliares y páncreas, en donde el común denominador es la indicación

\section{Correspondencia:}

*Yolanda Zamorano-Orozco 
correcta del procedimiento y que a la accesibilidad a la vía biliar sea óptima, además de otro parámetro de medición es la tasa de casos de pancreatitis post$\mathrm{CPRE}^{3}$. Uno de los puntos que más enfatizan en lo que respecta al ultrasonido endoscópico es la serie de eventos después de que se realiza un procedimiento terapéutico, como sangrado, perforación e infección.

La ergonomía es la parte de la economía que estudia la capacidad y psicología humanas en relación con el ambiente de trabajo y el equipo manejado por el trabajador. Fue definida por primera vez en 1961, en la revista internacional del trabajo, la aplicación conjunta de algunas ciencias biológicas y ciencias de la ingeniería para asegurar entre el hombre y el trabajo una óptima adaptación mutua con el fin de incrementar el rendimiento del trabajador y contribuir a su bienestar.

Desde el punto de vista ergonómico hay que distinguir dentro del sistema de trabajo dos aspectos:

- Aspecto técnico: No depende de la persona y se refiere a las características arquitectónicas y ambientales en las que se desarrolla la práctica laboral habitual. Luminosidad, temperatura, ventilación, accesibilidad al puesto de trabajo, disposición de cargas e instrumental.

- Aspecto humano: Análisis singular de la influencia del trabajo en la persona, gasto energético que requiere el trabajo, carga física implicada, nivel de atención requerido, carga mental, implicaciones en el aparato músculo esquelético, en el sistema cardiovascular, respiratorio, nervioso, etc.

En la realización de las endoscopias digestivas de las gastroscopias diagnósticas el tiempo aproximado de duración es de 5-10 minutos, aproximadamente 12 actuaciones en cada turno. En las terapéuticas de 3060 minutos por cada intervención; en las colonoscopias diagnósticas es de aproximadamente 30 minutos por intervención y en las terapéuticas aproximadamente entre 45-60 minutos por intervención.

En lo que respecta a radio-protección, la fluoroscopia es un elemento radiológico auxiliar en procedimientos terapéuticos de la vía biliar, páncreas, tracto gastrointestinal alto y bajo. Para el paciente, la fuente de exposición son los rayos $X$, se estima que los pacientes reciben alrededor de 2 a 16 minutos de fluoroscopia durante la CPRE, y que los procedimientos terapéuticos toman mucho más tiempo aún. Los estudios han hallado que durante una CPRE se usan valores del producto dosis área (PDA) de aproximadamente 13-66 Gy/cm2. Se han descrito dosis eficaces que van de 2 a 6 mSv por procedimiento ${ }^{4}$.

Para los endoscopistas y el resto del personal, la principal fuente de exposición a los rayos $X$ es la radiación que proviene del paciente, $y$ no el haz de rayos $X$ primario. Se han observado dosis eficaces promedio de alrededor de $0.07 \mathrm{mSv}$ por procedimiento para el caso de los endoscopistas que usan delantal de plomo. Si bien el cuerpo del endoscopista está bien protegido con el delantal de plomo, es posible que las partes de su cuerpo no protegidas reciban dosis importantes. Se han descrito dosis promedio en ojos de alrededor de 0.1-1.7 mGy por procedimiento y de alrededor de 0.5 mGy en manos. Las dosis que recibe el personal auxiliar habitualmente dependen de la posición y el tiempo que pasen cerca de la fuente de rayos $X$, ya que habitualmente se quedan parados más lejos del paciente. Los efectos de la radiación se dividen básicamente en dos categorías: los efectos deterministas, como la formación de cataratas, infertilidad, lesión cutánea, y caída de cabello; y los efectos estocásticos (cáncer y efectos genéticos).

Los efectos deterministas (principalmente cataratas y caída de cabello) han sido ya documentados en radiólogos y cardiólogos intervencionistas. No se han descrito efectos de ese tipo en gastroenterólogos. La cantidad de radiación que están usando los gastroenterólogos es relativamente pequeña, si se la compara con la que usan los radiólogos y los cardiólogos intervencionistas. El límite de dosis recomendado por la Comisión Internacional para la Protección Radiológica (International Commission on Radiological Protection: ICRP) y adoptada por la mayoría de los países es $20 \mathrm{mSv} / \mathrm{año}^{5}$. los dispositivos de protección personal (delantal con una equivalencia adecuada de plomo de $0.25-0.35 \mathrm{~mm}$ y del tipo envolvente, protector de tiroides, protector ocular, o protecciones para la cara/cabeza y región de miembros inferiores). Todos los puntos mencionados son una forma integral de los puntos mínimos y esenciales para cumplir con los estándares de calidad en la práctica de una endoscopia de excelencia cumplimento lo parámetros internacionales, así como normas de seguridad.

\section{Conflicto de intereses}

No existen conflictos de interés. Sin patrocinio de la industria.

\section{Bibliografía}

\footnotetext{
1. Adamas Megan. How Are quality measures developed by AGA? AGA, DDW, 2019.

2. Allen John, Wich quality metrics to track for colonoscopy and EGD. AGA, DDW 2019.

3. Riechiez Kermit. Quality in Colonoscopy. ASGE. DDW 2019.

4. Baron TH, Schueler BA. Pregnancy and radiation exposure during therapeutic ERCP: time to put the baby to bed? Gastrointest Endosc 2009;69:832-4.

5. International Atomic Energy Agency (IAEA). Dosimetry in diagnostic radiology: an international code of practice. (Technical Reports Series, no. 457, STI/DOC/010/457). Vienna: IAEA, 2007 [http://www-pub.iaea. org/MTCD/publications/PDF/TRS457_web.pdf].
} 


\title{
Indicadores de calidad en endoscopia superior: preparación, cromoendoscopia vital, digital
}

\author{
Omar Edel Trujillo-Benavides* \\ Hospital General de Zona 42, IMSS, Puerto Vallarta, Guadalajara, Jalisco, México
}

Palabras clave: Calidad. Seguridad intra y extrahospitalaria. Ergonomía. Radioprotección.

\section{Introducción}

En este capítulo se abordan los trabajos de investigación presentados en los foros internacionales "UEWG" 2018 y "DDW" 2019 sobre el tema de Indicadores de calidad de la esofagogastroduodenoscopia, enfocados en la preparación y el uso de la cromoendoscopia. Estos tópicos están incluidos dentro de los indicadores de calidad publicados por la Sociedad Americana de Endoscopia gastrointestinal (ASGE) 1 , y la Sociedad Europea de Endoscopia Gastrointestinal $(E S G E)^{2}$. Esta revisión incluye un resumen breve de algunos de estos trabajos.

En un estudio proveniente del Reino Unido se muestra que el uso del ácido acético para guiar la toma de biopsias en pacientes con esófago de Barrett para la detección de neoplasia (incluyendo displasia de bajo grado DBG, displasia de alto grado DAG y cáncer), denominado como protocolo de Portsmouth, comparado con el protocolo de Seattle es equiparable en cuanto a la detección de neoplasias, con la ventaja de ofrecer una disminución en número de biopsias tomadas (2,139 biopsias con protocolo de Seattle vs 226 biopsias con protocolo de Portsmouth), una disminución en los costos (125,897 Libras en protocolo de Seattle vs 13,311 Libras en protocolo de Portsmouth) y disminución en el número de biopsias por neoplasia detectada ( 357 vs 57 , respectivamente). Concluyendo que este protocolo no pasa inadvertida ninguna neoplasia, con la ventaja de disminuir el trabajo y los costos del análisis histológico ${ }^{3}$.

En un estudio realizado en Polonia, los autores comparan el uso de Imagen de Banda Estrecha (NBI, por sus siglas en inglés) contra la cromoendoscopia con Lugol para detectar neoplasias esofágicas en pacientes que recibieron tratamiento curativo para cáncer de cabeza y cuello. Los autores evalúan el valor predictivo positivo de las biopsias tomadas con la guía de ambos métodos, el número de biopsias tomadas, el tiempo de evaluación del esófago y la tolerancia al procedimiento. Ellos encuentran que el valor predictivo positivo fue similar para ambas estrategias $(7,69 \%$ para NBI (IC $95 \% 0.94 \%-25.1 \%$ ) y $8.11 \%$ para Lugol (IC 95\% 1.70-21.9\%) ( $p=1)$. Sin embargo, NBI superó al Lugol en el promedio de pacientes que requirió biopsias ( $12.7 \%$ vs $41.1 \%, p=0.003$ ), el tiempo de evaluación de esófago fue menor (3.5 min vs $5.15 \mathrm{~min}, \mathrm{p}$ $<0.001)$ y la tolerancia del procedimiento fue mejor. Concluyendo que el uso de NBI supera a la cromoendoscopia con Lugol en el número de biopsias requeridas, menor duración y mejor tolerancia del procedimiento, en la búsqueda de neoplasias esofágicas en población de alto riesgo; con un valor predictivo positivo similar4.

\section{Correspondencia:}


En México, el Dr. Cantú y un grupo de médicos del Hospital Dr. Carlos Mcgregor Sánchez del IMSS muestran sus resultados de una evaluación sobre la utilidad de la cromoendoscopia digital (Flexible Spectral Imaging Color Enhancement, FICE por sus siglas en inglés) para la detección de la metaplasia intestinal gástrica. Comparan el uso de la luz blanca con FICE para la toma guiada de biopsias, y nos muestran en sus resultados que el uso de FICE incrementa la sensibilidad, especificidad, los valores predictivo positivo y negativo (38.1\%, $97.1 \%$, $88.8 \%$ y $71 \%$ con FICE y $23.8 \%, 97.1 \%$ $83.3 \%$ y $67.3 \%$ con luz blanca respectivamente). Llegando a la conclusión de que el uso de FICE incrementa el promedio de detección de metaplasia intestinal gástrica comparado con el uso de luz blanca ${ }^{5}$.

El uso de la cromoendoscopia digital también ofrece un ahorro en el uso de recursos, como lo sugiere el Dr. R. Castro en un estudio donde evalúa la necesidad de separar las biopsias en diferentes frascos cuando la evaluación con NBI no muestra metaplasia intestinal gástrica. El estudio realizado en el Instituto Portugués de Oncología en Porto, Portugal, analiza en una serie de pacientes en quienes se tomo biopsia guiada por NBI y las muestras fueron colocadas en el mismo frasco y se determinó el puntaje OLGA y OLGIM: En sus resultados muestra la factibilidad del cálculo del sistema OLGA y OLGIM en todos los pacientes, así como la distinción de las muestras obtenidas de antro e incisura de aquellas obtenidas de cuerpo; sin tener implicaciones en el diagnóstico ni el seguimiento a pesar de colocar todas las muestras de cada paciente, en un mismo frasco. Concluye que este cambio en la práctica clínica diaria puede tener un impacto económico favorable ${ }^{6}$.

También se presentaron varios estudios en donde se evaluó el impacto de sistemas de ayuda por computadora e inteligencia artificial para el apoyo en la detección de neoplasias asociada a esófago de Barrett ${ }^{7}$, distinción de úlceras gástricas neoplásicas ${ }^{8}$, sistemas para mejorar la calidad de la evaluación endoscópica en tiempo real ${ }^{9}$, detección de gastritis activa asociada a infección por Helicobacter pylori ${ }^{10}$, el valor adicional que ofrece el uso de Imagen con Luz Azul (BLI por sus siglas en inglés) en la detección de neoplasias en pacientes con esófago de Barrett ${ }^{11}$.

Con el uso de NBI se ha logrado desarrollar y validar una nueva clasificación para la atrofia vellosa en pacientes con sospecha de enfermedad celiaca ${ }^{12}$, incluso el uso de NBI sin magnificación puede incrementar el promedio de detección de metaplasia intestinal, comparado con la evaluación con luz blanca convencional $^{13}$. Así como el diagnóstico de cáncer gástrico temprano en pacientes en quienes se ha erradicado el Helicobacter pylori ${ }^{14}$.

La mayoría de los estudios presentados sobre este tema evalúan la utilidad de herramientas diagnósticas relativamente nuevas 0 de reciente disponibilidad, sin embargo, son realizados con muestras pequeñas y requieren la replicación del análisis en otros estudios y poblaciones para contrastar los resultados. Un área de gran futuro es la del apoyo de inteligencia artificial en tiempo real que ayudaría a incrementar los promedios de detección de lesiones premalignas o malignas de manera temprana.

En esta ocasión no se presentaron estudios que abordaran los temas sobre la preparación de los pacientes para una esofagogastroduodenoscopia, como son indicaciones por escrito apropiadas sobre el ayuno previo a la endoscopia, la frecuencia con la cual se indican antibióticos de manera profiláctica en los escenarios en donde están indicados, frecuencia con la cual se documenta el plan de sedación, frecuencia con la cual se formula el manejo antitrombótico y se documenta antes del procedimiento, frecuencia con la cual se realiza una pausa del equipo antes de iniciar el procedimiento; todas estas son áreas de oportunidad que podrían abordarse en el futuro.

En conclusión, los estudios muestran una tendencia que favorece el uso de la cromoendoscopia digital sobre la cromoendoscopia vital; se observan buenos resultados de los sistemas de inteligencia artificial para el auxilio en tiempo real para la detección oportuna de lesiones. Queda pendiente la evaluación de indicadores preprocedimiento para establecer su utilidad real.

El autor declara no tener conflictos de interés ni haber recibido financiamiento para la elaboración de este manuscrito.

\section{Conflicto de intereses}

No existen conflictos de interés. Sin patrocinio de la industria.

\section{Bibliografía}

1. Park WG, Shaheen NJ, Cohen J, Pike IM, Adler DG. Quality indicators for EGD. Gastrointest Endosc 2015;81:17-30.

2. Bisschops R, Areia M, Coron E, et al. Performance measures for upper gastrointestinal endoscopy: A European Society of Gastrointestinal Endoscopy quality improvement initiative. United European Gastroenterology Journal 2016;4(5):629-656.

3. Longcroft-Wheaton L, Fogg C, De Caestecker J, et al. Acetic acid guided biopsies versus mapping biopsies for Barrett's surveillance: The ABBA study. United european gastroenterology Journal 2018;6(8S) A39.

4. Chaber-Ciopinska A, Kaminski MF, Wieszczy $P$, et al. Narrow Band Imaging endoscopy versus lugol chromoendoscopy in screening for esopjageal cancer among patients with head and neck cancers: a randomized trial. Gastrointest Endosc 2019;89(6S) AB93. 
5. Cantu Llanos E, Zamorano-Orozco Y, Ramírez-Ramírez MA, et al. Utility of digital chromoendoscopy (flexible spectral imaging color enhancement) FICE for the detection of gastric intestinal metaplasia. Gastrointest Endosc 2019;89(6S) AB360.

6. Castro R, Esposito G, Dinis-Ribeiro M, et al. And when NBI doesn't show gastric intestinal metaplasia: do we still need to separate biopsy samples into different vials? United European Gastroenterology Journal 2018;6 (8S) A179.

7. De Groof J, van der Sommen F, van der Putten J, et al. The ARGOS project: Computer aided detection system can detect Barrett neoplasia on endoscopic images with high accuracy. United European Gastroenterology Journal 2018;6(8S) A304.

8. Namikawa K, Hirasawa T, Ikenoyama $\mathrm{Y}$, et al. Can artificial intelligence-based diagnostic system perform differential diagnosis of gastric cancer and gastric ulcer. Gastrointest Endosc 2019;89(6S) AB74.

9. Yu HG, Wu L. Randomized controlled trial of wisense, a real time quality improving system for monitoring blind spots during esofagogastroduodenoscopy. Gastrointest Endosc 2019;89(6S) AB74.
10. Ono S, Dohi O, Yagi N, et al. Accuracies of endoscopic diagnostic of $\mathrm{H}$ pylori gastritis -Multicenter prospective study using white light imaging an linked color imaging-. Gastrointest endosc 2019;89(6S) AB654.

11. De Groof J, Swager A-F, Pouw R, et al. Blue Light imaging has an additional value to white light endoscopy in visualization or early Barrett's neoplasia. An international multicenter cohort study.United European Gastroenterology Journal 2018;6(8S) A305.

12. Gulati S, Emmanuel A, Pavlidis P, et al. Near focus NBI Classification of villous atrophy in suspected coeliac disease: International develompment an validation. Gastrointest Endosc 2019;89(6S): AB49.

13. Luk SW, Ng EK, Lau JY, et al. Randomized trial comparing white light endoscopy against non-magnifying narrow band imaging for upper $\mathrm{Gl}$ endoscopy. Gastrointest Endosc 2019;89(6S) AB163.

14. Tahara T, Horiguchi N, Terada T, et al. Real-time diagnostic yield of white light endoscopy, chromoendoscopy and magifying endoscopy with narrow band imaging in the undiagnosed gastric lesions after Helicobacter pylori eradication. Gastrointest Endosc 2019;89(6S) AB491. 